\author{
磷脂修饰化的石墨烯纳米复合物的制备及其直接电化学研究

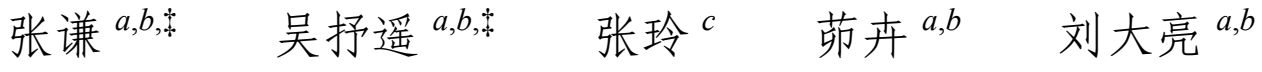 \\ 刘洋 $d$ 曾向群 $e$ 宋溪明*,a,b 李景虹 $*, d$ \\ ( ${ }^{a}$ 辽宁大学绿色合成与先进材料制备化学辽宁省重点实验室 沈阳 110036) \\ ( ${ }^{b}$ 辽宁大学化学院 沈阳 110036) \\ (c沈阳师范大学化学与生命科学学院 沈阳 110034) \\ ( ${ }^{d}$ 清华大学化学系 北京 100084) \\ ( 奥克兰大学化学系 罗切斯特市 美国)
}

\begin{abstract}
摘要 通过非共价键修饰方法制备了兼具良好生物相容性和导电性的磷脂 1-棕榈酰-2-油酰甘油-3-磷酸钠-石墨烯 (POPG-GP)纳米复合物, 并利用 TEM, FT-IR, UV-vis, zeta 电位等对其形貌和结构进行了表征. 由于 POPG-GP 纳米复合 材料在水溶液中呈现出较为明显的负电性, 因此, 可通过静电自组装方法将辣根过氧化物酶(HRP)进一步组装到 POPG-GP 修饰的玻碳(POPG-GP/GC)电极上，构筑 HRP/POPG-GP/GC 修饰电极. 电化学实验结果表明, POPG-GP 纳米 复合物能够有效实现 HRP 与修饰电极之间的直接电子转移. 此外, 固载在修饰电极表面的 HRP 对底物 $\mathrm{H}_{2} \mathrm{O}_{2}$ 还表现出 良好的电催化活性. HRP/POPG-GP/GC 修饰电极对 $\mathrm{H}_{2} \mathrm{O}_{2}$ 的检测线性范围为 $3.5 \sim 210 \mu \mathrm{mol} / \mathrm{L}$, 最低检出限为 $1.17 \mu \mathrm{mol} / \mathrm{L}$ $(S / N=3)$, 灵敏度为 $356.6 \mathrm{~mA} \cdot \mathrm{cm}^{-2} \cdot \mathrm{M}^{-1}, K_{\mathrm{m}}$ 值为 $0.45 \mathrm{mmol} / \mathrm{L}$.
\end{abstract}

关键词 石墨烯; 磷脂; 纳米复合物; 辣根过氧化物酶; 直接电化学

\title{
Biocompatible Phospholipid Modified Graphene Nanocomposite for Direct Electrochemistry of Redox Enzyme
}

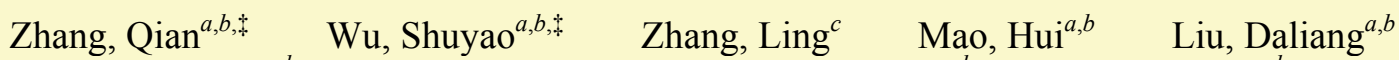 \\ Liu, Yang $^{d} \quad$ Zeng, Xiangqun ${ }^{e} \quad$ Song, Ximing*,a,b Li, Jinghong*,d \\ ( ${ }^{a}$ Liaoning Provincial Key Laboratory for Green Synthesis and Preparative Chemistry of Advanced Materials, \\ Liaoning University, Shenyang 110036) \\ ( ${ }^{b}$ College of Chemistry, Liaoning University, Shenyang 110036) \\ ( ${ }^{c}$ College of Chemistry and Life Science, Shenyang Normal University, Shenyang 110034) \\ ( ${ }^{d}$ Department of Chemistry, Tsinghua University, Beijing 100084) \\ ( ${ }^{e}$ Department of Chemistry, Oakland University, Rochester USA)
}

Abstract A novel lipid based carbonaceous nanocomposite, 1-palmitoyl-2-oleoyl-sn-glycero-3-phospho-(1'-rac-glycerol) (sodium salt) (POPG) modified graphene (GP) (POPG-GP), was designed and synthesized by a non-covalent method. The nanocomposite was endowed with excellent properties of the two independent components, such as the biocompatibility of POPG and the outstanding electric properties of graphene. Fourier transform infrared (FT-IR) spectra, ultraviolet-visible (UV-vis) absorption spectra, transmission electron microscopy (TEM) were utilized to characterize the structure, morphology and surface property of the as synthesized POPG-GP. It has been found that the modification of POPG on GP could not only assist the dispersion of graphene in aqueous solution, but also endow it with negatively charged, which was favorable for the further immobilization of model enzyme via self-assembly. Based on the electrostatic interaction, the positively charged horseradish peroxide (HRP) could be immobilized onto the surface of POPG-GP to form HRP/POPG-GP/GC electrode. UV-vis and FT-IR spectroscopies were used to monitor the assembly process and demonstrated that HRP had been immobilized without denaturation. The HRP/POPG-GP/GC electrode could commendably realize the direct electron transfer (DET) between electrode and redox enzyme with good electrochemical performance. Moreover, such modified electrode also

\footnotetext{
*E-mail: jhli@mail.tsinghua.edu.cn, songlab@Inu.edu.cn *为共同第一作者

Received August 30, 2013; published November 3, 2013.

Project supported by the National Natural Science Foundation of China (Nos. 21235004, 21128005, 51273087, 21071070, 21203126, 21005046, 51203072, 20901035), the Financial Supports from the Program for Liaoning Innovative Research Team in University (No. LT2011001), the Natural Science Foundation of Liaoning Province (Nos. 201202088, LJQ2013112), the Research Fund for the Doctoral Program of Liaoning Province (No. 20131042), the Foundation for Young Scholars of Liaoning University (Nos. 2012LDQN08, 2012LDQN07), College students' innovative entrepreneurial training program (No. X201210140036) and the Foundation of 211 Project for Innovative Talents Training, Liaoning University.

项目受国家自然科学基金(Nos. 21235004, 21128005, 51273087, 21071070, 21203126, 21005046, 51203072, 20901035)、辽宁省高等学校创新团队基金 (No. LT2011001)、辽宁省自然科学基金(Nos. 201202088, LJQ2013112)、辽宁省博士科研启动基金项目(No. 20131042)、辽宁大学青年科研基金(Nos. 2012LDQN08, 2012LDQN07)、辽宁大学创新创业训练计划项目(No. X201210140036)和辽宁大学创新人才培养基金资助.
} 
showed good electrocatalytic response toward the detection of $\mathrm{H}_{2} \mathrm{O}_{2}$ with high sensitivity, wide linear range, excellent stability and reproducibility. The linear response range for the HRP/POPG-GP/GC was $3.5 \sim 210 \mu \mathrm{mol} / \mathrm{L}(R=0.999)$. The detection limit and the sensitivity of the HRP/POPG-GP/GC electrode was calculated to be $1.17 \mu \mathrm{mol} / \mathrm{L}(S / N=3)$ and 356.6 $\mathrm{mA} \cdot \mathrm{cm}^{-2} \cdot \mathrm{M}^{-1}$, respectively. The apparent Michaelis-Menten constant $K_{\mathrm{m}}$ was estimated to be $0.45 \mathrm{mmol} / \mathrm{L}$, indicating a high affinity of HRP to $\mathrm{H}_{2} \mathrm{O}_{2}$ on POPG-GP. The experiment results demonstrated that POPG-GP not only provided a biocompatible microenvironment for the immobilized HRP, but also supplied a necessary pathway for its direct electron transfer. Therefore, such biocompatible nanocomposite had potential applications in the field of biosensors.

Keywords graphene; phospholipid; nanocomposite; horseradish peroxide; direct electrochemistry

\section{1 引言}

利用电化学方法研究生物氧化还原酶的直接电子 转移, 对了解生命体的能量转换和物质代谢、生物大分 子的结构与物理化学性质的关系、以及探索其在生命体 内的电子传递过程的机理和生理作用都具有重要的理 论指导意义 ${ }^{[1,2]}$. 然而, 由于大部分氧化还原酶在电极表 面会产生强烈的吸附, 从而使其构象发生改变, 甚至失 去生物活性，进而抑制了它们在电极上的电子传递 ${ }^{[3 \sim 5]}$. 而且, 酶的电活性中心往往被多肽链包围, 从而阻碍了 其在电极表面的直接电子转移 ${ }^{[5 \sim 8]}$. 因此, 为了实现氧 化还原酶在电极表面发生可逆或准可逆的直接电子转 移反应，选择合适的酶固载方法和材料甚为重要.

作为二维的碳基纳米材料, 石墨烯具有比表面积 大、导电性好等诸多优良特性 ${ }^{[9,10]}$. 作为电极材料, 石墨 烯已在电化学领域, 尤其是生物电化学领域受到了高度 的关注, 并得到了广泛应用 ${ }^{[11,12]}$. 近期研究表明, 石墨 烯良好的电学性质可有效地促进氧化还原酶或蛋白质 与电极间的直接电子传输, 并提高生物传感器的性能. 然而, 由于石墨烯片层间存在较强的范德华力和 $\pi-\pi$ 相 互作用, 易发生不可逆的团聚, 从而丧失其单层二维纳 米片的结构特性 ${ }^{[13,14]}$. 此外, 石墨烯的表面还呈现一定 的疏水性, 这一方面使其难以有效分散在极性或非极性 的溶剂中, 另一方面还妨碍了其与水溶性生物大分子的 进一步作用, 限制了石墨烯在生物电化学中的应用. 近 期研究表明, 通过对石墨烯进行适宜的功能化修饰, 不 但可提高其溶解性以及在不同基质中的分散性, 还可由 此为石墨烯引入特定的功能性组分, 赋予石墨烯更为独 特的性质.

磷脂(Phospholipid) 是分子结构中含有磷酸的脂质 (lipid). 作为生物膜的重要组成部分, 磷脂具有极好的 生物相容性, 并已被广泛地应用于酶固载, 仿生材料和 DNA 转染等诸多生物相关的研究领域. Dayani 等 ${ }^{[15]}$ 利 用共价修饰方法将 1,2-二硬酯酸-3-磷脂酰乙醇胺 (DPPE)修饰于碳纳米管表面, 制备了新型碳基纳米材 料. 研究结果表明, 脂质分子的表面修饰不仅可有效提 高碳基材料的生物相容性, 还能改善其在不同极性溶剂 中的分散性. 然而目前此类相关研究仍较为有限, 尤其 对于二维的石墨烯而言, 其与脂质的相互作用以及应用 方面的相关研究鲜有报道.

本文首次将具有良好生物相容性的磷脂 1-棕榈酰- 2-油酰甘油-3-磷酸钠(1-palmitoyl-2-oleoyl-sn-glycero3-phospho-(1'-rac-glycerol) (sodium salt), POPG)与石墨 烯通过非共价修饰方法复合, 制备了兼具良好生物相容 性和较好导电性的石墨烯纳米复合物(POPG-GP). 在此 基础上，利用 POPG-GP 复合物的表面电荷性质，通过 静电组装方法实现了辣根过氧化物酶(HRP)在复合物上 的固载, 并构建了 HRP/POPG-GP/GC 修饰电极. 该修饰 电极不仅能够较好实现固载的 HRP 与电极表面间的直 接电子传输, 还对 $\mathrm{H}_{2} \mathrm{O}_{2}$ 表现出优良的电催化性能.

\section{2 结果与讨论}

\subsection{POPG-GP 纳米复合物的制备及其修饰电极的构 筑}

图 1 为 POPG-GP 纳米复合物的制备及其修饰电极 构筑过程的示意图. 首先, 通过 Hummers 方法 ${ }^{[16]}$ 制备氧 化石墨烯(GO). 进而, 在 POPG 水溶液中, 利用 $\mathrm{N}_{2} \mathrm{H}_{4} \cdot \mathrm{H}_{2} \mathrm{O}$ 还原 $\mathrm{GO}$. 由图 1 可知, POPG 分子具有两亲 性，其疏水的烃基链和亲水的极性基团各处于分子的两 端. 因此在还原过程中, POPG 的烃基链会通过疏水作 用吸附于石墨烯表面，而极性基团则朝向外部，与水亲 和. 这种非共价表面修饰不但可使原位生成的石墨烯表 现出较好的水溶性, 还使其在水溶液中呈现负电荷特 性. 由于辣根过氧化物酶(HRP)的等电点为 $8.9^{[17]}$, 其在 $\mathrm{pH}$ 值低于等电点的水溶液中呈现正电荷. 基于此, 在 pH 5.7 的 PBS 中, HRP 即可通过静电吸附的相互作用负 载于 POPG-GP 表面, 实现 HRP/POPG-GP/GC 修饰电极 的构筑. 这种基于自组装构建修饰电极的方法可以使酶 在温和条件下固载到石墨烯的表面，从而有效地保持固 载酶的天然结构和生物电活性.

\subsection{POPG-GP 复合材料的形貌表征}

图 2A 为 POPG-GP 纳米复合物的 TEM 图. 从图中 可以清晰地观察到 POPG-GP 呈明显的薄纱状且带有褶 皱、蜷曲的片状结构. 图 2B 为 POPG-GP 纳米复合物在 水中分散液的照片. 由图可知, 由于 POPG 的表面修饰, 石墨烯呈现出较好的水溶性, 从而能够在水中形成均一 的黑色分散液. POPG 修饰化的石墨烯分散液较为稳定, 放置 3 个月后无明显的沉降产生.

图 $2 \mathrm{C}$ 为 POPG-GP 纳米复合物在 $\mathrm{pH} 5.7$ 的 PBS 中 的 zeta 电位图. 测试结果表明 POPG-GP 纳米复合物在 


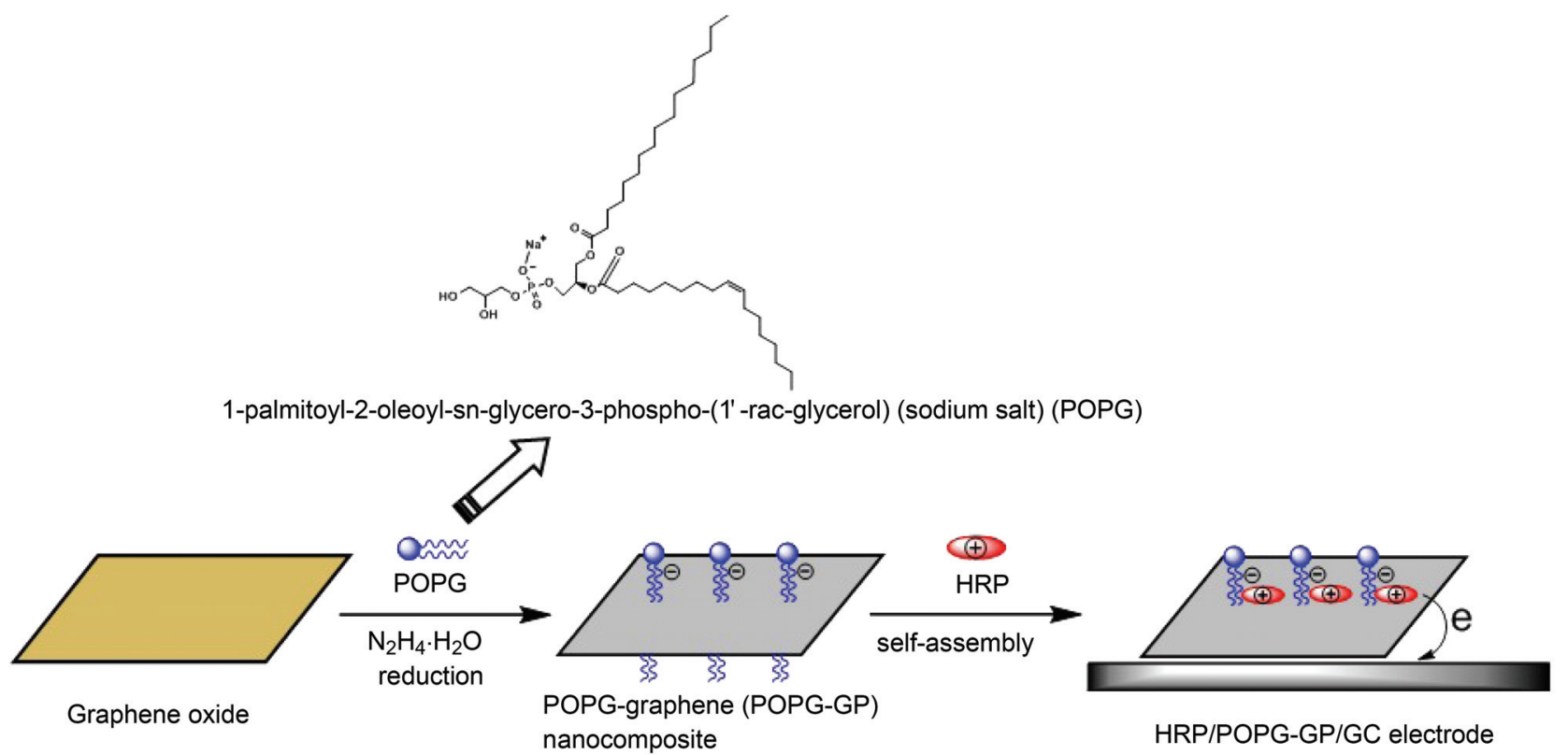

图 $1 \mathrm{HRP} / \mathrm{POPG}-\mathrm{GP} / \mathrm{GC}$ 修饰电极构筑过程示意图

Figure 1 Fabrication of HRP/POPG-GP/GC electrode and the direct electron transfer between HRP and glassy carbon (GC) electrode
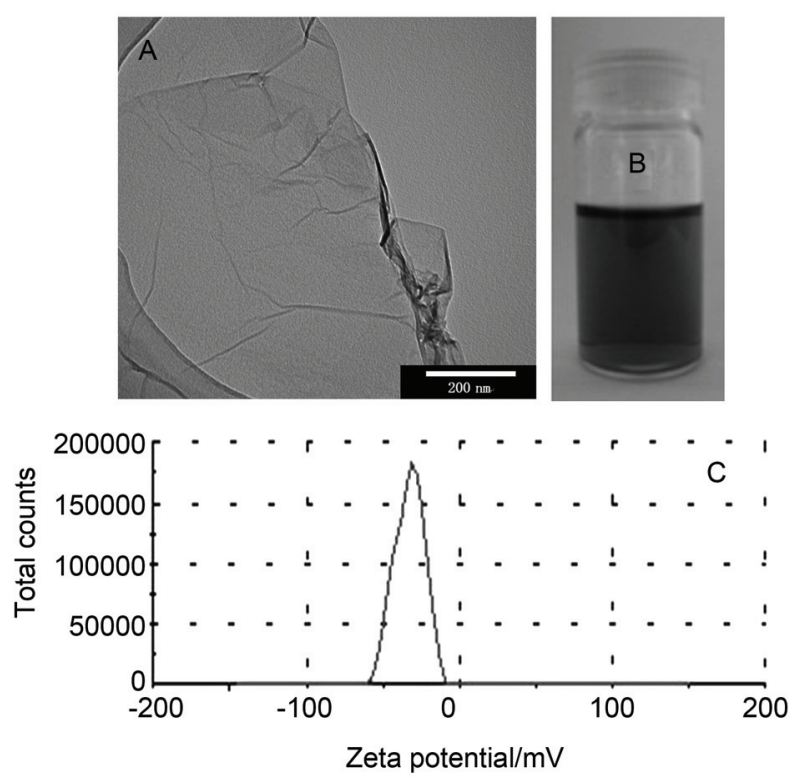

图 2 (A) POPG-GP 的 TEM 图, (B) POPG-GP 分散在水中的照片和(C) POPG-GP 在 PBS (pH 5.7) 中的 zeta 电位图

Figure 2 (A) TEM image, (B) photo and (C) zeta potential image of POPG-GP

PBS 中呈现出较强的电负性, 其 zeta 电位值约为 -32.9 $\mathrm{mV}$. 石墨烯表面的这种电荷性质不但可使纳米片间存 在较强的静电排斥作用，从而有效防止石墨烯片间的不 可逆聚集, 而且还为其进一步通过静电吸附方法固载氧 化还原酶提供了条件.

\subsection{POPG-GP 复合材料光谱表征}

图 3 为 GO (a)和 POPG-GP (b) 在溶液中的 UV-vis 光谱图. 由图可知, GO 的特征吸收峰位于 $231 \mathrm{~nm}$ (图 3a), 而 POPG-GP 的特征吸收峰则红移到 $267 \mathrm{~nm}$ 处(图 $3 b)$. 这种红移现象的产生是由于水合肼的还原过程促 使氧化石墨烯恢复了部分 $\pi-\pi$ 键, 从而扩大了其共轭体 系所造成的.

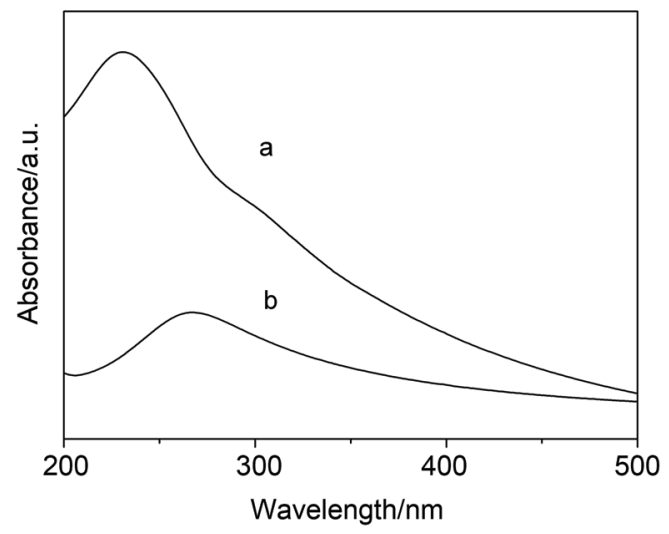

图 3 (a) GO 和(b) POPG-GP 水溶液的 UV-vis 图

Figure 3 UV-vis absorption spectra of (a) GO and (b) POPG-GP in water

\subsection{HRP/POPG-GP 纳米复合物的光谱表征}

图 4 分别为 POPG-GP (a), HRP/POPG-GP (b), HRP (c)的 FT-IR 光谱谱图. 由曲线 a 可观察到 POPG-GP 的 谱图中分别存在 $1627 \mathrm{~cm}^{-1}$ 处石墨烯 $\mathrm{C}=\mathrm{C}$ 的伸缩振动 峰和 $1735 \mathrm{~cm}^{-1}$ 处修饰磷脂的 $\mathrm{C}=\mathrm{O}$ 的伸缩振动峰. 而 在 HRP/POPG-GP 的谱图中, 不但可观察到与 POPG-GP 类似的特征吸收峰, 还可观察到 HRP 的酰胺 I 带(1650 $\left.\mathrm{cm}^{-1}\right)$ 和酰胺 II 带 $\left(1543 \mathrm{~cm}^{-1}\right)$ 的特征吸收峰. 这些固载 酶的特征吸收峰与 HRP 谱图中的特征吸收峰(图 4c)基 本一致. HRP/POPG-GP 在 $1650 \mathrm{~cm}^{-1}$ 处的吸收带较 HRP 
的有所展宽, 这是由于 POPG-GP 与 HRP 的特征吸收峰 互相重叠所致. 由此可知, 固载于 POPG-GP 上的 HRP 有效保持了其固有的二级结构.

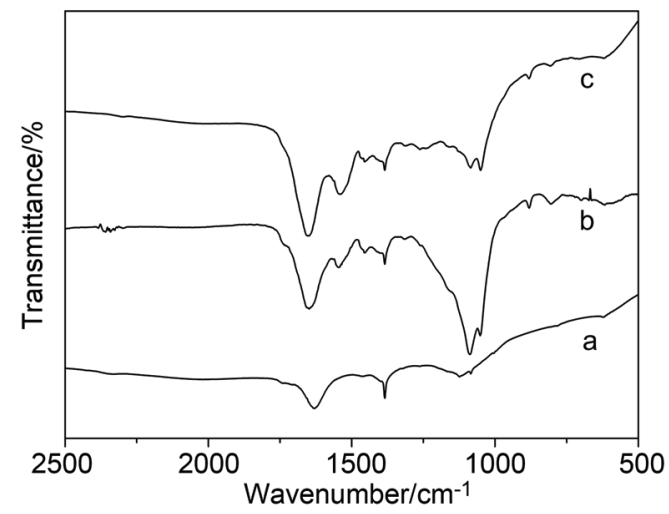

图 4 (a) POPG-GP, (b) HRP/POPG-GP 和(c) HRP 的 FT-IR 图 Figure 4 FT-IR spectra of (a) POPG-GP, (b) HRP/POPG-GP and (c) HRP

图 5 为 POPG-GP (a), HRP/POPG-GP (b) 和 HRP (c) 在 PBS 中的 UV-vis 光谱图. 由曲线 $\mathrm{a}$ 可知, POPG-GP(图 5 a) 在 $350 \sim 500 \mathrm{~nm}$ 的检测范围内没有明显的特征吸收 峰. 而当其在 $\mathrm{pH} 5.7$ 的 PBS 中与 HRP 相互作用后, 其 UV-vis 谱图中(图 5b)在 $403 \mathrm{~nm}$ 处出现了明显的吸收峰. 这与 HRP(图 5c)谱图中的 Soret 带的特征吸收峰完全一 致. 由此可说明, 一方面 POPG 的表面修饰有效促进了 酶在石墨烯表面的负载; 另一方面, 由于固载条件较为 温和, 而且石墨烯纳米复合物具有较好的生物相容性, 固载的 HRP 的生物活性得到了很好地保持.

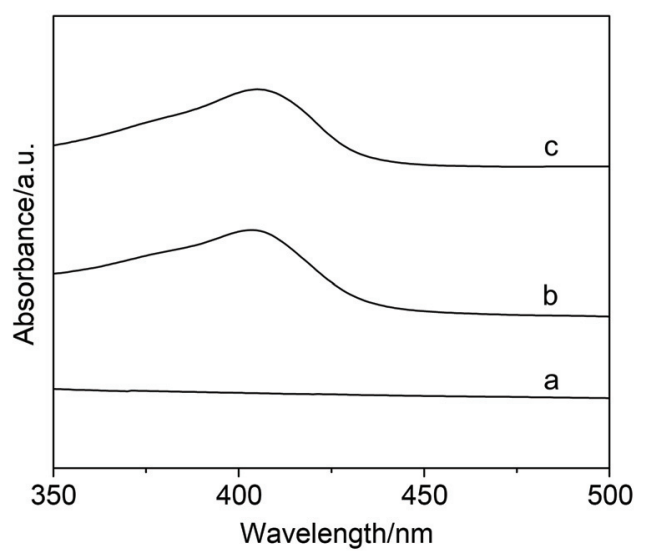

图 5 (a) POPG-GP, (b) HRP/POPG-GP 和(c) HRP 在 $0.1 \mathrm{~mol} / \mathrm{L}$ PBS $(\mathrm{pH}$ 5.7)中的 UV-vis 图

Figure 5 UV-vis absorption spectra of (a) POPG-GP, (b) HRP/POPG-GP and (c) HRP in $0.1 \mathrm{~mol} / \mathrm{L}$ PBS (pH 5.7)

\section{$2.5 \mathrm{HRP} / \mathrm{POPG}-\mathrm{GP} / \mathrm{GC}$ 修筑电极的直接电化学性质}

图 6 为 $\mathrm{HRP} / \mathrm{POPG}-\mathrm{GP} / \mathrm{GC}$ 和 $\mathrm{POPG}-\mathrm{GP} / \mathrm{GC}$ 修饰电

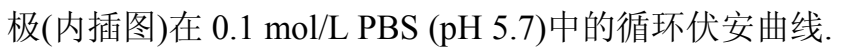
图 6 内插图中, 没有观察到 POPG-GP/GC 修饰电极氧化
还原峰的出现，说明 POPG-GP 在扫描范围内没有电化 学活性. 而 HRP/POPG-GP/GC 修饰电极的循环伏安图 中可观察到一对明显且可逆的氧化还原峰. 其氧化峰 $\left(E_{\mathrm{pa}}\right)$ 电位为 $-0.211 \mathrm{~V}$, 还原峰电位 $\left(E_{\mathrm{pc}}\right)$ 为 $-0.320 \mathrm{~V}$, 式 量电位为 $E^{0}=-0.266 \mathrm{~V}$. 这是由固载酶的 $\mathrm{HRP}-\mathrm{Fe}^{\mathrm{III}} / \mathrm{Fe}^{\mathrm{II}}$ 电对在修饰电极表面发生了直接电子转移而产生的, 与 文献报道的 HRP 修饰电极的检测结果基本一致 ${ }^{[18]}$. 由 以上分析可知，由于 POPG-GP 纳米复合物兼具较好的 生物相容性和导电性，因此可有效促进负载 HRP 与玻 碳(GC)电极表面间的直接电子转移.

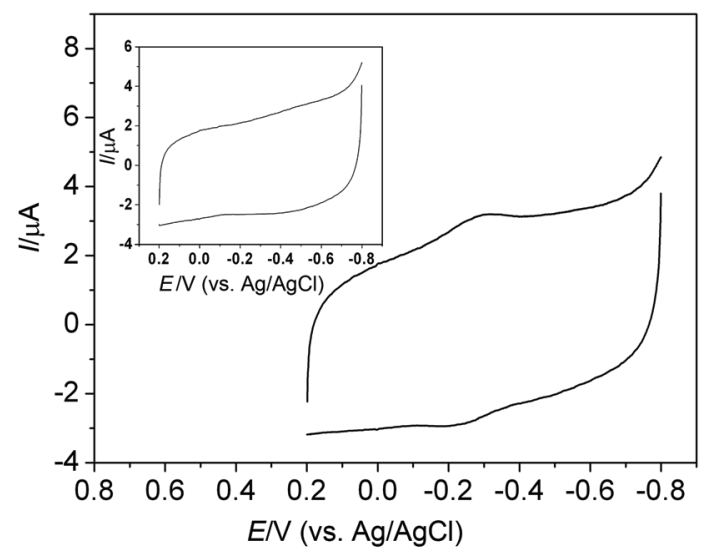

图 $6 \mathrm{HRP} / \mathrm{POPG}-\mathrm{GP} / \mathrm{GC}$ 修饰电极在 $\mathrm{N}_{2}$ 饱和的 $0.1 \mathrm{~mol} / \mathrm{L}$ PBS (pH 5.7) 中的循环伏安图, 扫速: $200 \mathrm{mV} \cdot \mathrm{s}^{-1}$. 内插图: POPG-GP/GC 修饰电极 Figure 6 Cyclic voltammograms of POPG-GP/GC (Inset) and $\mathrm{HRP} / \mathrm{POPG}-\mathrm{GP} / \mathrm{GC}$ electrodes in $\mathrm{N}_{2}$-saturated $0.1 \mathrm{~mol} / \mathrm{L} \mathrm{PBS}(\mathrm{pH} 5.7)$. Scan rate, $200 \mathrm{mV} \cdot \mathrm{s}^{-1}$

利用循环伏安法还考察了 HRP/POPG-GP/GC 修饰 电极的氧化还原峰电流与扫速间的相关性. 图 7 为修饰 电极在不同扫速下的循环伏安图. 如图所示, 当扫速增 加时，其氧化峰电流和还原峰电流同时增加. 根据法拉 第方程 $Q=n F A \Gamma^{*}(Q$ ：反应消耗的电量, 可由积分 $\mathrm{HRP}$ 的还原峰面积得到, $n$ 和 $F$ 分别为转移的电子数和法拉 第常数, $A$ 为电极的几何面积, $\Gamma^{*}$ 为电极表面电活性 HRP 的表面覆盖度), 可求算电化学活性的 HRP 在修饰 电极的 $\Gamma^{*}$ 约为 $3.62 \times 10^{-11} \mathrm{~mol} \cdot \mathrm{cm}^{-2}$. 由于 HRP 最大表 面单层覆盖度的理论值为 $2.0 \times 10^{-11} \mathrm{~mol} \cdot \mathrm{cm}^{-2[19]}$, 因此 可说明 HRP 是以多层的状态负载于修饰电极上的. 这 是由 POPG-GP 纳米复合物具有的较大比表面积以及较 好导电性所造成的. 图 7 内插图为 HRP/POPG-GP/GC 修饰电极在不同扫速时氧化还原峰电流相对扫速的线 性关系图. 由图可知, 扫速从 100 到 $800 \mathrm{mV} \cdot \mathrm{s}^{-1}$ 的范围 内, 修饰电极的峰电流与扫速成正比, 并且呈现出较好 的线性关系，说明电极反应为表面控制过程.

\section{$2.6 \mathrm{pH}$ 值对 HRP/POPG-GP/GC 修饰电极的电化学 行为的影响}

酶的生物活性只有在较为温和的条件下才能够得 以较好的保持. 在影响酶生物活性的诸多条件中, 溶液 


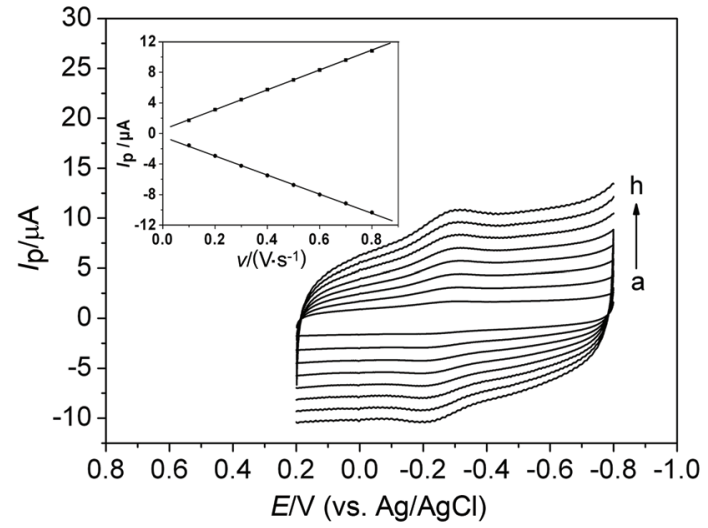

图 $7 \mathrm{HRP} / \mathrm{POPG}-\mathrm{GP} / \mathrm{GC}$ 修饰电极在 $0.1 \mathrm{~mol} / \mathrm{L} \mathrm{PBS}(\mathrm{pH}$ 5.7) 中, 不同 扫速下的循环伏安曲线, 从 $\mathrm{a}$ 到 $\mathrm{h}: 100,200,300,400,500,600,700$, $800 \mathrm{mV} \cdot \mathrm{s}^{-1}$. 内插图: 氧化还原峰电流相对扫速的线性图

Figure 7 Cyclic voltammograms of HRP/POPG-GP/GC electrode at scan rates of 100, 200, 300, 400, 500, 600, 700 and $800 \mathrm{mV} \cdot \mathrm{s}^{-1}$ (from a to h) in $0.1 \mathrm{~mol} / \mathrm{L}$ PBS (pH 5.7), respectively; Plots of oxidation peak current $(\bullet)$ and reduction peak current $(\mathbf{\square})$ vs. scan rate (Inset)

的 $\mathrm{pH}$ 值是一个重要的因素. 溶液 $\mathrm{pH}$ 值的改变会直接影 响酶活性中心催化基团的解离, 从而影响酶与底物之间 的结合. 只有在适宜的 $\mathrm{pH}$ 条件下, 酶反应才可达到最 大的速度. 基于此, 本文考察了溶液 $\mathrm{pH}$ 值对 $\mathrm{HRP} /$ POPG-GP/GC 修饰电极电化学行为的影响. 图 $8 \mathrm{~A}$ 为 $\mathrm{HRP} / \mathrm{POPG}-\mathrm{GP} / \mathrm{GC}$ 修饰电极的还原峰电流与溶液 $\mathrm{pH}$ 值 间的关系图. 由图可知, 修饰电极在溶液 $\mathrm{pH}$ 值为 5.7 时 呈现出最大还原峰电流响应. 此外, 由图 $8 \mathrm{~B}$ 还可观察 到 HRP/POPG-GP/GC 修饰电极的式电位与溶液 $\mathrm{pH}$ 值之 间存在较好的线性关系, 其斜率为 $-44.9 \mathrm{mV} / \mathrm{pH}$. 该结 果表明固载于修饰电极表面的 HRP 的电子传输为质子 耦合过程 ${ }^{[20]}$.

\subsection{HRP/POPG-GP/GC 修饰电极的电化学催化性能}

图 9A 为 $\mathrm{HRP} / \mathrm{POPG}-\mathrm{GP} / \mathrm{GC}$ 修饰电极在 $0.1 \mathrm{~mol} / \mathrm{L}$ PBS (pH 5.7) 中对不同浓度 $\mathrm{H}_{2} \mathrm{O}_{2}$ 电化学催化的循环伏安 图. 由图可知, 在无氧的情况下, 当缓冲溶液中加入 $\mathrm{H}_{2} \mathrm{O}_{2}$ 后, 修饰电极的循环伏安曲线发生了显著的形变. 其位于 $-0.3 \mathrm{~V}$ 附近的还原峰电流明显增加, 而氧化峰 电流则明显减小. 随着 $\mathrm{H}_{2} \mathrm{O}_{2}$ 浓度的逐渐增大, 修饰电极 的催化还原峰电流逐渐增大, 氧化峰电流逐渐消失, 说 明在 $\mathrm{HRP} / \mathrm{POPG}-\mathrm{GP} / \mathrm{GC}$ 修饰电极上, 固载的 HRP 不仅 能够实现直接电子转移, 而且还对 $\mathrm{H}_{2} \mathrm{O}_{2}$ 的还原表现出 良好的电化学催化活性. HRP 对 $\mathrm{H}_{2} \mathrm{O}_{2}$ 催化过程的机理 如下所示 ${ }^{[21]}$ :

$$
\begin{aligned}
& \mathrm{HRP}[\mathrm{Heme}(\mathrm{FeIII})]+\mathrm{H}_{2} \mathrm{O}_{2} \rightarrow \text { Compound } \mathrm{I}+\mathrm{H}_{2} \mathrm{O} \\
& \text { Compound } \mathrm{I}+\mathrm{e}^{-} \rightarrow \text { Compound II } \\
& \text { Compound } \mathrm{II}+\mathrm{e}^{-} \rightarrow \mathrm{HRP}[\text { Heme(FeIII)] }
\end{aligned}
$$

图 9B 是 HRP/POPG-GP/GC 修饰电极的催化电流与 $\mathrm{H}_{2} \mathrm{O}_{2}$ 浓度之间的线性关系曲线. 从图中可以观察到 $\mathrm{H}_{2} \mathrm{O}_{2}$ 浓度在 3.5 210 $\mu \mathrm{mol} / \mathrm{L}$ 范围内与阴极峰电流表现
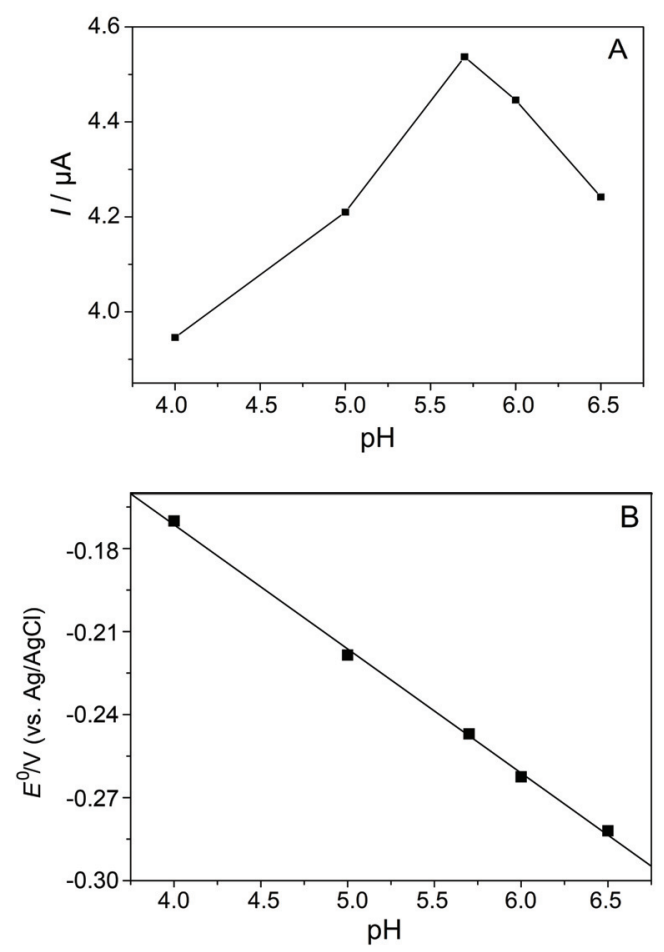

图 8 (A) HRP/POPG-GP/GC 修饰电极在 $\mathrm{pH} 4.0 \sim 6.5$ 的 $\mathrm{PBS}$ 中的循 环伏安曲线峰电流与 $\mathrm{pH}$ 值关系图, 扫速: $200 \mathrm{mV} \cdot \mathrm{s}^{-1}$; (B) 式电位 $E^{0}$ 与 $\mathrm{pH}$ 值的关系图

Figure 8 (A) The influence of $\mathrm{pH}$ on the response of the $\mathrm{HRP} / \mathrm{POPG}-\mathrm{GP} / \mathrm{GC}$ electrode in $0.1 \mathrm{~mol} / \mathrm{L}$ PBS. Scan rate, $200 \mathrm{mV} \cdot \mathrm{s}^{-1}$; (B) the dependence of the formal potential of the HRP/POPG-GP/GC electrode vs. $\mathrm{pH}$

出良好的线性关系, 其线性回归方程为: $y=0.033 x+$ $0.059(R=0.999, n=12), y$ 和 $x$ 分别代表还原峰电流 $(\mu \mathrm{A})$ 和 $\mathrm{H}_{2} \mathrm{O}_{2}$ 的浓度 $(\mu \mathrm{mol} / \mathrm{L})$. 经对比, 该修饰电极的催化线 性范围比已报道的其它类型 HRP 修饰电极的相关值宽 很多 ${ }^{[22]}$. 根据线性方程可知, HRP/POPG-GP/GC 修饰电 极对 $\mathrm{H}_{2} \mathrm{O}_{2}$ 检测的灵敏度和最低检测限分别为 356.6 $\mathrm{mA} \cdot \mathrm{cm}^{-2} \cdot \mathrm{M}^{-1}$ 和 $1.17 \mu \mathrm{mol} / \mathrm{L}(S / N=3)$. 此外, 由图 $9 \mathrm{~B}$ 还可观察到随着 $\mathrm{H}_{2} \mathrm{O}_{2}$ 浓度的增加, 修饰电极的催化电 流与 $\mathrm{H}_{2} \mathrm{O}_{2}$ 浓度间的线性关系出现了一个平台，该现象 符合酶催化的 Michaelis-Menten 反应特征. 根据 Lineweaver-Burk 方程 $\left(1 / I_{\mathrm{ss}}=1 / I_{\max }+K_{\mathrm{m}} /\left(I_{\max } C\right)\right)\left(I_{\mathrm{ss}}\right.$, 稳 态响应电流; $I_{\max }$, 底物饱和时最大稳态电流; $C$, 底物浓 度; $K_{\mathrm{m}}$, 表观米式常数)可知 ${ }^{[23]}$, 固载酶的 $K_{\mathrm{m}}$ 为 0.45 $\mathrm{mmol} / \mathrm{L}$, 远小于已报道的 HRP 修饰电极上的 $K_{\mathrm{m}}$ 值 ${ }^{[24,25]}$. 较小的 $K_{\mathrm{m}}$ 表明固载于 POPG-GP 上的 $\mathrm{HRP}$ 与底物 $\mathrm{H}_{2} \mathrm{O}_{2}$ 间存在较好的亲和力, 使修饰电极表现出较好的电化学 催化性能.

\section{$2.8 \mathrm{HRP} / \mathrm{POPG}-\mathrm{GP} / \mathrm{GC}$ 修饰电极的重复性和稳定性}

通过循环伏安法对 HRP/POPG-GP/GC 修饰电极的 重现性和稳定性进行了考察. 由 5 支相同的玻碳电极所 制备的 HRP/POPG-GP/GC 修饰电极, 分别对 $30 \mu \mathrm{mol} / \mathrm{L}$ 

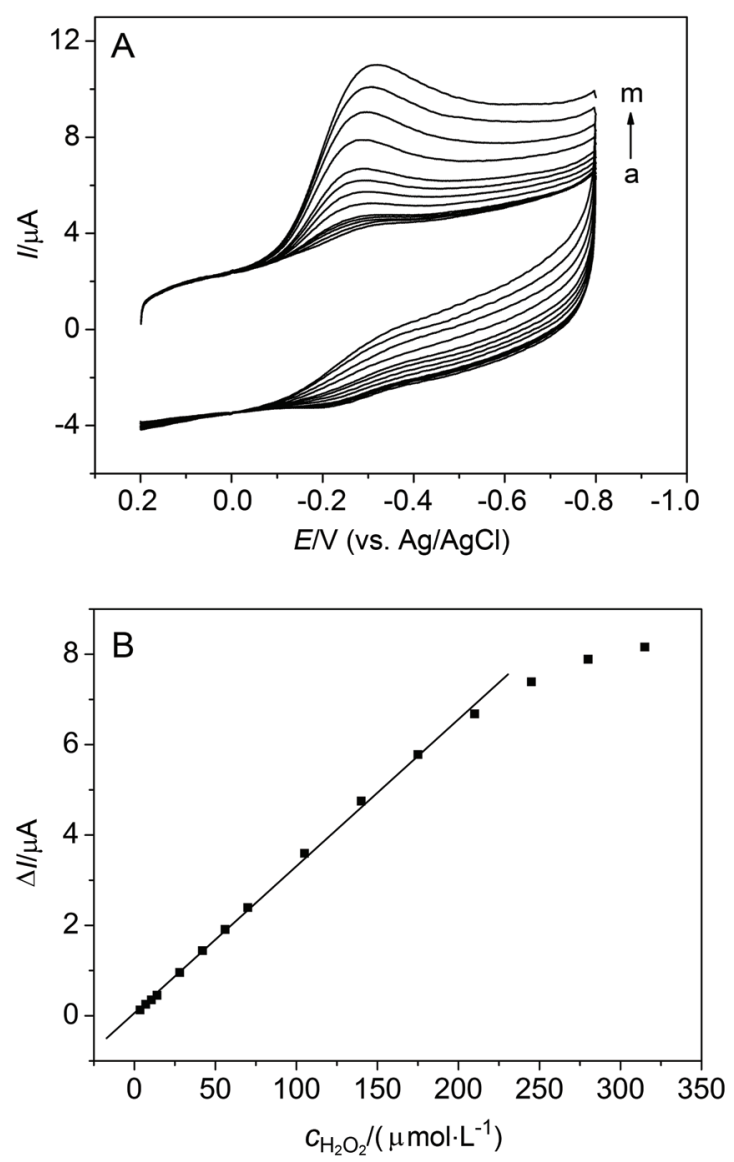

图 9 (A) $\mathrm{HRP} / \mathrm{POPG}-\mathrm{GP} / \mathrm{GC}$ 修饰电极在 $0.1 \mathrm{~mol} / \mathrm{L} \mathrm{PBS}(\mathrm{pH}$ 5.7)中, 对 $\mathrm{H}_{2} \mathrm{O}_{2}$ 催化的循环伏安曲线, 扫速: $200 \mathrm{mV} \cdot \mathrm{s}^{-1}$. $\mathrm{H}_{2} \mathrm{O}_{2}$ 的浓度: (a) 0 , (b) 3.5, (c) 7, (d) 10.5, (e) 14, (f) 28, (g) 42, (h) 56, (i) 70, (j) 105, (k) 140, (1) 175 和 (m) $210 \mu \mathrm{mol} / \mathrm{L}$; (B) 还原峰电流与 $\mathrm{H}_{2} \mathrm{O}_{2}$ 浓度的校准曲线 Figure 9 (A) Cyclic voltammograms of HRP/POPG-GP/GC electrode in $0.1 \mathrm{~mol} / \mathrm{L}$ PBS (pH 5.7) containing (a) 0, (b) 3.5, (c) 7, (d) 10.5 , (e) 14 , (f) 28 , (g) 42, (h) 56, (i) 70, (j) 105, (k) 140, (l) 175 , and (m) $210 \mu \mathrm{mol} / \mathrm{L}$ $\mathrm{H}_{2} \mathrm{O}_{2}$. Scan rate, $200 \mathrm{mV} \cdot \mathrm{s}^{-1}$; (B) Plots of the electrocatalytic current $(\Delta I)$ vs. $\mathrm{H}_{2} \mathrm{O}_{2}$ concentration

$\mathrm{H}_{2} \mathrm{O}_{2}$ 的循环伏安测试结果可知, 修饰电极的电流响应 值的相对标准偏差为 $3.2 \%$, 说明了该修饰电极具有较 好的重现性.

为了研究 $\mathrm{HRP} / \mathrm{POPG}-\mathrm{GP} / \mathrm{GC}$ 修饰电极的稳定性, 分别将修饰电极浸泡在 pH 5.7 的 PBS 中放入 $4{ }^{\circ} \mathrm{C}$ 冰箱 中储存 $12 \mathrm{~h}$ 以及 $7 \mathrm{~d}$ 后考察其对 $30 \mu \mathrm{mol} / \mathrm{L} \mathrm{H}_{2} \mathrm{O}_{2}$ 循环伏 安的检测. 如图 10 所示, 修饰电极在放置 $12 \mathrm{~h}$ 以及 7 天后, 其循环伏安曲线的阴极峰电流均没有明显变化. 该结果表明 HRP/POPG-GP/GC 修饰电极对 $\mathrm{H}_{2} \mathrm{O}_{2}$ 的电催 化具有较好的稳定性, 这可归因于 POPG-GP 纳米复合 材料良好的生物相容性.

\section{3 结论}

本文首次利用非共价键修饰方法制备了 POPG-GP 纳米复合物, 并进一步利用静电自组装的方法实现了 $\mathrm{HRP}$ 在纳米复合物上的固载, 构筑了 $\mathrm{HRP} / \mathrm{POPG}-\mathrm{GP} /$

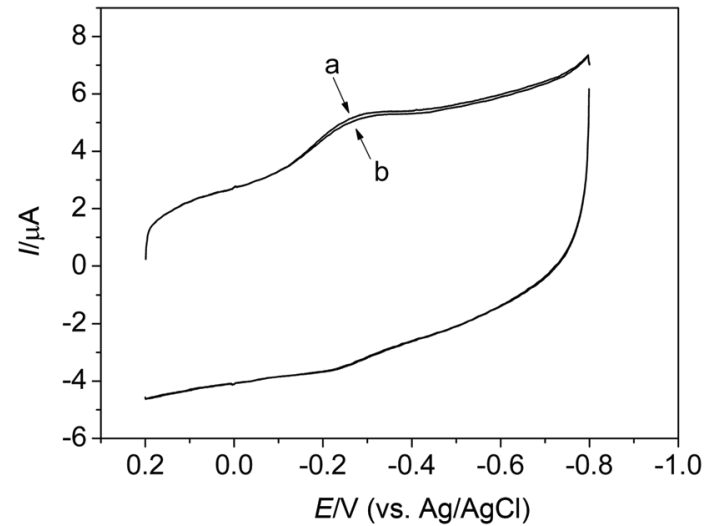

图 $10 \mathrm{HRP} / \mathrm{POPG}-\mathrm{GP} / \mathrm{GC}$ 修饰电极放置 $12 \mathrm{~h}$ 和 $7 \mathrm{~d}$ 后, 在 $0.1 \mathrm{~mol} / \mathrm{L}$ PBS (pH 5.7)中, 对 $30 \mu \mathrm{mol} / \mathrm{L} \mathrm{H}_{2} \mathrm{O}_{2}$ 催化的循环伏安曲线, 扫速: 200 $\mathrm{mV} \cdot \mathrm{s}^{-1}$

Figure 10 Cyclic voltammograms of $\mathrm{HRP} / \mathrm{POPG}-\mathrm{GP} / \mathrm{GC}$ electrode in $0.1 \mathrm{~mol} / \mathrm{L} \mathrm{PBS} \mathrm{(pH} \mathrm{5.7)} \mathrm{with} 30 \mu \mathrm{mol} / \mathrm{L} \mathrm{H}_{2} \mathrm{O}_{2}$ after stored for $12 \mathrm{~h}$ (a) and for 7 days (b). Scan rate, $200 \mathrm{mV} \cdot \mathrm{s}^{-1}$

$\mathrm{GC}$ 修饰电极. 由于 POPG-GP 纳米复合物兼具较好的生 物相容性和导电性, 固载的 HRP 不但可以保持其天然 的结构, 还可实现与玻碳电极表面间的直接电子转移. 此外, HRP/POPG-GP/GC 修饰电极还对 $\mathrm{H}_{2} \mathrm{O}_{2}$ 的电化学 催化表现出较好的性能, 因此有望被应用于生物传感器 及燃料电池等领域.

\section{4 实验部分}

\section{1 试剂}

1-棕榈酰-2-油酰甘油-3-磷酸钠(POPG)(日本 NOF 公司), 辣根过氧化物酶(HRP)(Sigma 公司), 水合肼 $\left(\mathrm{NH}_{2}-\mathrm{NH}_{2} \cdot \mathrm{H}_{2} \mathrm{O}\right)$ 、铁氰化钾、磷酸二氢钠、磷酸氢二钠(国 药集团化学试剂有限公司), $30 \%$ 过氧化氢 $\left(\mathrm{H}_{2} \mathrm{O}_{2}\right)$ (天津市 博迪化工有限公司), 其它试剂均为分析纯. 本实验用 水为超纯水.

\subsection{POPG-GP 纳米复合材料的制备}

氧化石墨烯(GO)采用 Hummers 法制备 ${ }^{[16]}$. 将 $4 \mathrm{mg}$ $\mathrm{GO}$ 分散到 $4 \mathrm{~mL}$ 水中, 加入 $8 \mathrm{mg}$ POPG, 漩浴混合, 使 $\mathrm{POPG}$ 均匀分散到 $\mathrm{GO}$ 溶液中, 然后加入 $28 \mu \mathrm{L}$ 水合肼. $80{ }^{\circ} \mathrm{C}$ 下, 反应 $4 \mathrm{~h}$ 得到黑色均一的溶液. 将产物用 0.22 $\mu \mathrm{m}$ 的滤膜过滤，用水洗涤溶液至中性，最后将制得的 POPG-GP 配成 $0.1 \mathrm{mg} / \mathrm{mL}$ 的水溶液.

\subsection{HRP/POPG-GP/GC 修饰电极的制备}

将直径为 $3 \mathrm{~mm}$ 的玻碳电极进行预处理: 首先, 将 电极放到抛光布上用粒径分别为 $1.0,0.3,0.05 \mu \mathrm{m} \mathrm{Al}_{2} \mathrm{O}_{3}$ 粉末进行抛光, 蒸馏水冲洗, 然后分别用丙酮、乙醇、 超纯水超声清洗. 最后用高纯氮气吹干, 室温下放置备 用.

取 $5 \mu \mathrm{L} 0.1 \mathrm{mg} / \mathrm{mL}$ 的 POPG-GP 纳米复合物滴涂于 玻碳电极表面，室温下干燥成膜. 然后，将修饰电极浸 入到用 $0.1 \mathrm{~mol} / \mathrm{L}$ (pH 5.7) PBS 配制的 HRP (5 mg/mL) 溶 
液中, $4{ }^{\circ} \mathrm{C}$ 下放置约 $24 \mathrm{~h}$, 最终得到 $\mathrm{HRP} / \mathrm{POPG}-\mathrm{GP} / \mathrm{GC}$ 修饰电极.

\section{4 仪器设备}

石墨烯基纳米复合材料利用 H-800 透射电镜显微镜 (TEM)(日本 Hitachi 公司), L35-900 紫外可见光谱仪 (UV-vis)(美国 Perkin-Elmer 公司), Spectrum One 红外光 谱仪(FT-IR)(美国 Perkin-Elmer 公司), Nano ZS 纳米粒度 及 zeta 电位分析仪(英国 Malvern 公司)进行表征.

电化学实验利用 BAS100B 电化学工作站(美国 Bioanalytical Systems 公司)进行表征. 本实验电化学测 试采用三电极体系: 铂丝电极为对电极, $\mathrm{Ag} / \mathrm{AgCl}$ 电极 为参比电极, $P O P G-G P / G C$ 或 $H R P / P O P G-G P / G C$ 电极为 工作电极. 测试底液为 $8 \mathrm{~mL} \mathrm{pH} 5.7$ 浓度为 $0.1 \mathrm{~mol} / \mathrm{L}$ 的 磷酸缓冲溶液(PBS), 通入高纯氮气 $30 \mathrm{~min}$, 以除去溶 液中的溶解氧. 若无特殊说明, 在实验过程中测试杯内 一直保持氮气饱和氛围.

\section{References}

[1] Kang, X.; Wang, J.; Wu, H.; Aksay, I. A.; Liu, J.; Lin, Y. Biosens. Bioelectron. 2009, 25, 901 .

[2] Liu, Y.; Wang, M.; Zhao, F.; Xu, Z.; Dong, S. Biosens. Bioelectron. $\mathbf{2 0 0 5}, 21,984$

[3] Chen, H.; Dong, S. Biosens. Bioelectron. 2007, 22, 1811.

[4] Lu, X.; Hu, J.; Yao, X.; Wang, Z.; Li, J. Biomacromolecules 2006, 7, 975.

[5] Dai, Z.; Ju, H. Acta Phys.-Chim. Sin. 2004, 20, 1262. (戴志晖, 鞠熀 先, 物理化学学报, 2004, 20, 1262.)
[6] Lu, X.; Zhang, Q.; Zhang, L.; Li, J. Electrochem. Commun. 2006, 8, 874.

[7] Zhang, Q.; Wu, S.; Zhang, L.; Lu, J.; Verproot, F.; Liu, Y.; Xing, Z.; Li, J.; Song, X.-M. Biosens. Bioelectron. 2011, 26, 2632.

[8] Cai, C.; Chen, J. Acta Chim. Sinica 2004, 62, 335. (蔡称心, 陈静, 化学学报, 2004, 62, 335.)

[9] Novoselov, K. S.; Geim, A. K.; Morozov, S. V.; Jiang, D.; Katsnelson, M. I.; Grigorieva, I. V.; Dubonos, S. V.; Firsov, A. A. Nature 2005, 438, 197.

[10] Li, D.; Muller, M. B.; Gilje, S.; Kaner, R. B.; Wallace, G. G. Nat. Nanotechnol. 2008, 3, 101.

[11] Zhang, Q.; Yang, S.; Zhang, J.; Zhang, L.; Kang, P.; Li, J.; Xu, J.; Zhou, H.; Song, X.-M. Nanotechnology 2011, 22, 494010.

[12] Zhang, Q.; Wu, S.; He, M.; Zhang, L.; Liu, Y.; Li, J.; Song, X.-M. Acta Chim. Sinica 2012, 70, 2213. (张谦, 吴抒遥, 何茂伟, 张玲, 刘洋, 李景虹, 宋溪明, 化学学报, 2012, 70, 2213.)

[13] Niyogi, S.; Bekyarova, E.; Itkis, M. E.; McWilliams, J. L.; Hamon, M. A.; Haddon, R. C. J. Am. Chem. Soc. 2006, 128, 7720.

[14] Yang, H.; Zhang, Q.; Shan, C.; Li, F.; Han, D.; Niu, L. Langmuir 2010, 26, 6708

[15] Dayani, Y.; Malmstadt, N. Langmuir 2012, 28, 8174.

[16] Hummers, W. S.; Offeman, R. E. J. Am. Chem. Soc. 1958, 80, 1339.

[17] Wang, Z.; Li, M.; Su, P.; Zhang, Y.; Shen, Y.; Han, D.; Ivaska, A.; Niu, L. Electrochem. Commun. 2008, 10, 306.

[18] Li, J.; Dong, S. J. Electroanal. Chem. 1997, 431, 19.

[19] Zhang, L.; Zhang, Q.; Lu, X.; Li, J. Biosens. Bioelectron. 2007, 23, 102.

[20] Bond, A. M.; Braun, R. D. J. Electrochem. Soc. 1980, 127, 528C.

[21] Zhang, Q.; Qiao, Y.; Hao, F.; Zhang, L.; Wu, S.; Li, Y.; Li, J.; Song, X. M. Chem. Eur. J. 2010, 16, 8133.

[22] Mohammed, E.; Ignacio, N.-R.; Manuel, D.; Maria, P. H.-A.; Dolores, B.-M.; Jose, L. H.-H. C. Electrochim. Acta 2008, 53, 7131.

[23] Kamin, R. A.; Wilson, G. S. Anal. Chem. 1980, 52, 1198.

[24] Zhao, X.; Mai, Z.; Kang, X.; Zou, X. Biosens. Bioelectron. 2008, 23, 1032 .

[25] Zhou, K.; Zhu, Y.; Yang, X.; Luo, J.; Li, C.; Luan, S. Electrochim. Acta 2010, 55, 3055. 